\title{
Investigation of Structural Phase Transition of PbS
}

\author{
Purvee Bhardwaj \\ High Pressure Research Laboratory, Department of Physics, Barkatullah University, Bhopal 462026, India
}

Correspondence should be addressed to Purvee Bhardwaj, purveebhardwaj@gmail.com

Received 29 August 2012; Accepted 11 October 2012

Academic Editors: J. Jeffries, M. Kato, K. Matsuhira, and L. Pusztai

Copyright () 2012 Purvee Bhardwaj. This is an open access article distributed under the Creative Commons Attribution License, which permits unrestricted use, distribution, and reproduction in any medium, provided the original work is properly cited.

The high-pressure structural phase transition of semiconductor PbS has been investigated, using the three body potential (TBP) model. Phase transition pressures are associated with a sudden collapse in volume. The phase transition pressures and related volume collapses obtained from this model show a generally good agreement with available results. Moreover, the elastic properties of $\mathrm{PbS}$ are also investigated.

\section{Introduction}

In current years, IV-VI semiconductors are one of the most important narrow gap materials in electronics. These semiconductors are widely applied in fundamental information of solid-state electronic device fabrication. In this group of semiconductors, lead chalcogenides $\mathrm{PbX}(X=\mathrm{S}$, $\mathrm{Se}$, and $\mathrm{Te}$ ) are primarily semiconducting materials [1]. Lead chalcogenides are well known to be good materials for thermoelectric due to their low thermoconductivity. Lead chalcogenides remain one of the basic materials of modern infrared optoelectronics [2].

The structural high pressure behaviour of lead chalcogenides has paid considerable interest from both theoretical and experimental works [3-10]. Among these lead chalcogenides the lead sulphide $\mathrm{PbS}$ is useful as detector in infrared radiation. $\mathrm{PbS}$ crystallizes in the rocksalt $(\mathrm{NaCl})$ type $\left(B_{1}\right)$ structure at normal condition. First-order phase transition of $\mathrm{PbS}$ has been studied using high-pressure X-ray diffraction [4-7]. The ab initio electronic study of pressure induced structural phase transition of lead sulphide has been carried out by Bencherif et al. [8]. Phase transformation and conductivity in nanocrystal $\mathrm{PbS}$ under pressure have been investigated by Jiang et al. [9]. Zhang et al. investigated the elastic properties of $\mathrm{PbS}$ [10]. First-principles study of $B_{1}$ to $B_{2}$ phase transition in $\mathrm{PbS}$ has been performed by Bhambhani et al. [11].

It seems from the above literature that the present compound is less studied; we applied the three-body potential
(TBP) model to the present compound for studying the highpressure phase transition and volume collapse. The need of inclusion of three-body interaction forces was emphasized by many workers for the betterment of results $[12,13]$. The model and calculation is given in Section 2 and the result and discussion is given in Section 3.

\section{Model and Calculation}

Application of pressure directly results in compression leading to the increased charge transfer (or three body interaction effect [14]) due to the deformation of the overlapping electron shell of the adjacent ions (or nonrigidity of ions) in solids.

These effects have been included in the Gibbs free energy $(G=U+P V-T S)$ as a function of pressure and three body interactions (TBI) [14], which are the most dominant among the many body interactions. Here, $U$ is the internal energy of the system equivalent to the lattice energy at temperature near zero, $P$ is pressure, $V$ is volume, and $S$ is the entropy. The Gibbs free energies for rock salt $\left(B_{1}\right)$ and $\mathrm{CsCl}\left(B_{2}\right)$ structures at temperature $T=0 \mathrm{~K}$ are given by the following equations:

$$
\begin{gathered}
G_{B 1}(r)=U_{B 1}(r)+P V_{B 1}(r), \\
G_{B 2}\left(r^{\prime}\right)=U_{B 2}\left(r^{\prime}\right)+P V_{B 2}\left(r^{\prime}\right) .
\end{gathered}
$$

With $V_{B 1}\left(=2.00 r^{3}\right)$ and $V_{B 2}\left(=1.54 r^{\prime 3}\right)$ as unit cell volumes for $B_{1}$ and $B_{2}$ phases, respectively. The first terms in (1) 
are lattice energies for $B_{1}$ and $B_{2}$ structures and they are expressed as follows:

$$
\begin{aligned}
U_{B_{1}}(r)= & \frac{-\alpha_{m} z^{2} e^{2}}{r}-\frac{\left(12 \alpha_{m} z e^{2} f(r)\right)}{r}-\left[\frac{C}{r^{6}}+\frac{D}{r^{8}}\right] \\
& +6 b \beta_{i j} \exp \left[\frac{\left(r_{i}+r_{j}-r\right)}{\rho}\right] \\
& +6 b \beta_{i i} \exp \left[\frac{\left(2 r_{i}-1.414 r\right)}{\rho}\right] \\
& +6 b \beta_{j j} \exp \left[\frac{\left(2 r_{j}-1.414 r\right)}{\rho}\right], \\
U_{B_{2}}\left(r^{\prime}\right)= & \frac{-\alpha_{m}^{\prime} z^{2} e^{2}}{r^{\prime}}-\frac{\left(16 \alpha_{m}^{\prime} z e^{2} f\left(r^{\prime}\right)\right)}{r^{\prime}}-\left[\frac{C^{\prime}}{r^{\prime} 6}+\frac{D^{\prime}}{r^{\prime} 8}\right] \\
& +8 b \beta_{i j} \exp \left[\frac{\left(r_{i}+r_{j}-r^{\prime}\right)}{\rho}\right] \\
& +3 b \beta_{i i} \exp \left[\frac{\left(2 r_{i}-1.154 r^{\prime}\right)}{\rho}\right] \\
& +3 b \beta_{j j} \exp \left[\frac{\left(2 r_{j}-1.154 r^{\prime}\right)}{\rho}\right] .
\end{aligned}
$$

Here, $\alpha_{m}$ and $\alpha_{m}^{\prime}$ as the Madelung constants for $\mathrm{NaCl}$ and $\mathrm{CsCl}$ structure, respectively. $C\left(C^{\prime}\right)$ and $D\left(D^{\prime}\right)$ are the overall van der Waal coefficients of $B_{1}\left(B_{2}\right)$ phases, $\beta_{i j}(i, j=1,2)$ are the Pauling coefficients. Ze is the ionic charge and $\mathrm{b}(\rho)$ are the hardness (range) parameters, $r\left(r^{\prime}\right)$ are the nearest neighbour separations for $\mathrm{NaCl}(\mathrm{CsCl})$ structure $f(r)$ is the three body force parameter.

These lattice energies consists of long-range Coulomb energy (first term), three-body interactions corresponding to the nearest neighbour separation $r\left(r^{\prime}\right)$ (second term), $\mathrm{vdW}$ (van der Waal) interaction (third term), and energy due to the overlap repulsion represented by Hafemeister and Flygare (HF) type potential and extended up to the second neighbour ions (remaining terms).

\section{Results and Discussion}

The input data of the crystal are listed in Table 1. The Gibbs free energies contain three-model parameters $(b, \rho, f(r))$. The values of these parameters have been estimated using the first- and second-order space derivatives of the lattice energy $(U)$ given as follows [15-20]:

$$
\begin{gathered}
{\left[\frac{d U}{d r}\right]_{r=r_{0}}=0,} \\
{\left[\frac{d^{2} U}{d r^{2}}\right]_{r=r_{0}}=9 k r_{0} B_{T} .}
\end{gathered}
$$

The values of computed model parameters have been given in Table 1. With these model parameters and the
TABLE 1: Input and model parameters for PbS.

\begin{tabular}{lccccc}
\hline \multirow{2}{*}{ Solid } & \multicolumn{2}{c}{ Input parameters } & \multicolumn{3}{c}{ Model parameters } \\
& $r_{0}(\AA)$ & $B_{T}(\mathrm{GPa})$ & $b\left(10^{-12} \mathrm{ergs}\right)$ & $\rho(\AA)$ & $f(\mathrm{r})$ \\
\hline $\mathrm{PbS}$ & $2.9645^{\mathrm{a}}$ & $62.2^{\mathrm{a}}$ & 4.5637 & 0.339 & 0.01479 \\
\hline${ }^{\mathrm{a}}$ Reference $[3]$. & & & &
\end{tabular}

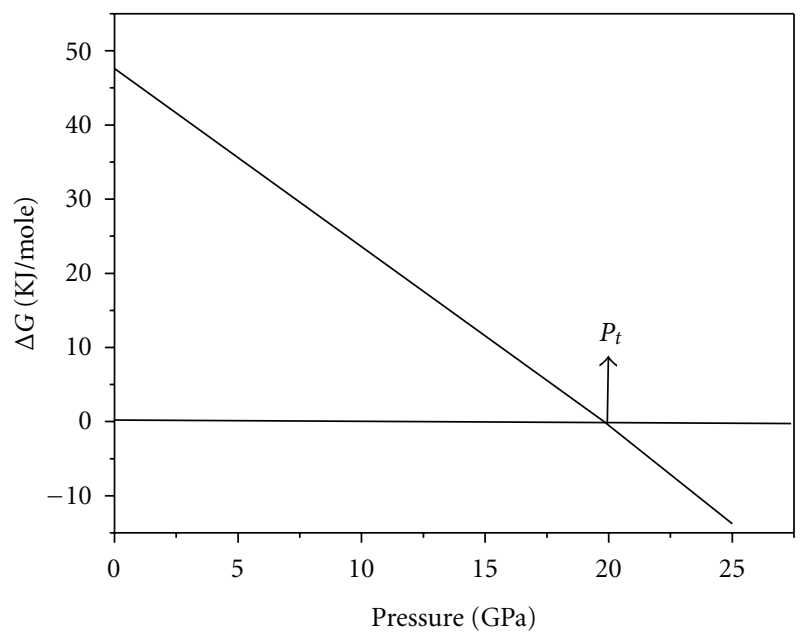

FIGURE 1: Variation of Gibbs free energy with pressure for PbS.

minimization technique, the phase transition pressure of the present compound has been computed.

3.1. Structural Properties. The present compound is stable in $\mathrm{NaCl}$ structure at normal condition and high pressure it transforms to body centre $\mathrm{CsCl}$ structure. We have followed the technique of minimization of Gibbs free energies of $\mathrm{NaCl}$ and $\mathrm{CsCl}$ phases. We have minimized $G_{B_{1}}(r)$ and $G_{B_{2}}\left(r^{\prime}\right)$ given by (2) at different pressures in order to obtain the interionic separations $r$ and $r^{\prime}$ corresponding to $B_{1}$ and $B_{2}$ phases associated with minimum energies. The phase transition pressure $\left(P_{t}\right)$ is the pressure at which $\Delta G$ approaches zero. At $P_{t}$ these compounds undergo a $\left(B_{1}-B_{2}\right)$ transition associated with a sudden collapse in volume. Figure 1 shows our present computed phase transition pressure for $\mathrm{NaCl}$ type to CsCl-type structures in $\mathrm{PbS}$ at $19.8 \mathrm{GPa}$. The present phase transition pressure pointed up by arrows in Figure 1. The value of phase transition pressure has been listed in Table 2 and compared with experimental [4] and other theoretical results [8]. It is remarkable to note from the Table 2 that the phase transition pressure $\left(P_{t}\right)$, obtained from present model is in closer agreement with experimental than others theoretical results [8].

In addition, the pressure volume behaviour of the present compound has also been studied. At the phase transition pressure there is a sudden collapse in volume has been occurred. This type of transition shows the first-order phase transition. The computed value of volume collapse for lead sulphide has been given in Table 2. The compression curve has been plotted in Figure 2 for $\mathrm{PbS}$. The $\mathrm{NaCl}$ and $\mathrm{CsCl}$ phases have been shown in this figure. 
TABLE 2: Phase transition and volume change of $\mathrm{PbS}$.

\begin{tabular}{|c|c|c|c|c|}
\hline \multirow{2}{*}{ Solid } & \multicolumn{3}{|c|}{ Phase transition pressure (GPa) } & Volume collapse $\%$ \\
\hline & Present & Expt. & Others & Present \\
\hline $\mathrm{PbS}$ & 19.8 & 21.5 & $25.3^{\mathrm{a}}, 8.13^{\mathrm{b}}$ & 5.63 \\
\hline
\end{tabular}

${ }^{\mathrm{a}}$ Reference [4], ${ }^{\mathrm{b}}$ reference [9].

3.2. Elastic Properties. Elastic constants supply useful information concerning with the change in the character of covalent and ionic forces induced in the crystal as it is subjected to the phase transformation. We have calculated the second-order elastic constants, bulk modulus, and pressure derivative of bulk modulus for lead sulphide. The information of second-order elastic constants (SOECs) and their pressure derivatives are important for the understanding of the interatomic forces in solids. The expressions of second order elastic constants are expressed as [14-19] follows:

$$
\begin{gathered}
C_{11}=\left(\frac{e^{2}}{4 a^{4}}\right)\left[-5.112 Z(Z+12 f(r))+A_{1}\right. \\
\left.+\frac{\left(A_{2}+B_{2}\right)}{2}+9.3204 z a f^{\prime}(r)\right], \\
C_{12}=\left(\frac{e^{2}}{4 a^{4}}\right)\left[0.226 Z(Z+12 f(r))-B_{1}\right. \\
\left.+\frac{\left(A_{2}-5 B_{2}\right)}{4}+9.3204 z a f^{\prime}(r)\right], \\
C_{44}=\left(\frac{e^{2}}{4 a^{4}}\right)\left[2.556 Z(Z+12 f(r))-B_{1}\right. \\
\left.+\frac{\left(A_{2}+3 B_{2}\right)}{4}\right] .
\end{gathered}
$$

Using model parameters $(b, \rho, f(r))$, pressure derivatives of bulk modulus have been computed whose expressions are as follows:

$$
\begin{gathered}
\frac{d B_{T}}{d p}=-(3 \Omega)^{-1} \\
\times\left[\begin{array}{c}
13.980 Z(Z+12 f(r))+C_{1}-3 A_{1}+C_{2}-3 A_{2} \\
-167.7648 z a f^{\prime}(r)+41.9420 z a^{2} f(r)
\end{array}\right], \\
B_{T}=\frac{1}{3}\left(C_{11}+2 C_{12}\right), \quad S=\frac{1}{2}\left(C_{11}-C_{12}\right), \\
\Omega=-2.330 Z(Z+12 f(r))+A_{1}+A_{2} \\
\quad+21.9612 z a f^{\prime}(r) .
\end{gathered}
$$

The values of $A_{i}, B_{i}$, and $C_{i}(i=1,2)$ have been evaluated from the knowledge of $b, \rho$, and vdW coefficients.

The calculated values of second order elastic constants and pressure derivative of bulk modulus have been given in Table 3. We have compared our results with experimental [7] and other theoretical results [10]. The present calculated values are in good agreement with experimental results than

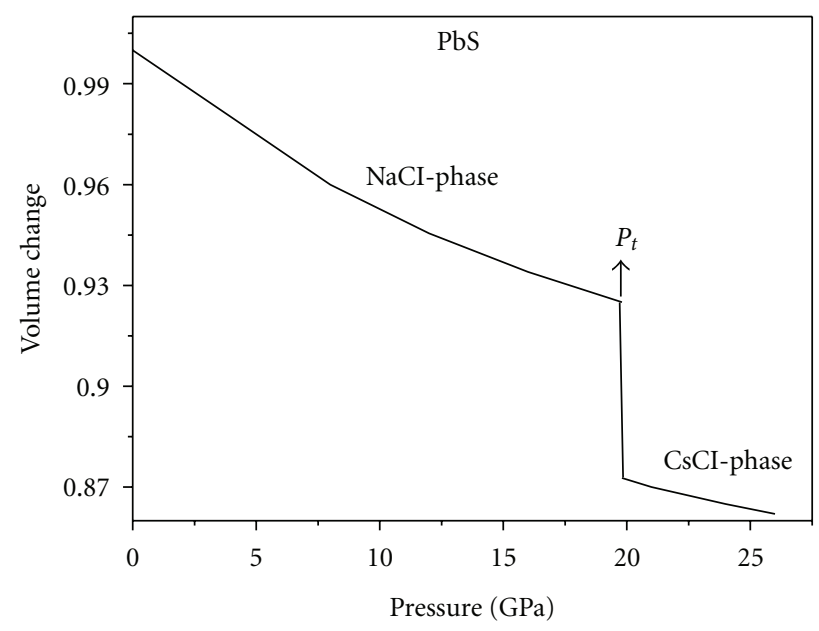

Figure 2: Variation of volume change $V_{P} / V_{0}$ with pressure for $\mathrm{PbS}$.

TABLE 3: Calculated values of elastic constants (in GPa) and pressure derivative of bulk modulus and Cauchy discrepancy $(\delta)$ of lead sulphide.

\begin{tabular}{lccc}
\hline Solid & Present & Experimental & Others \\
\hline$C_{11}$ & 131.62 & $124.0^{\mathrm{a}}$ & $135.1^{\mathrm{b}}$ \\
$C_{12}$ & 30.45 & $33.0^{\mathrm{a}}$ & $16.9^{\mathrm{b}}$ \\
$C_{44}$ & 19.86 & $23.0^{\mathrm{a}}$ & $20.4^{\mathrm{b}}$ \\
$d B_{T} / d P$ & 3.2 & - & $3.8^{\mathrm{c}}$ \\
$\delta$ & 10.59 & 10 & -3.5 \\
$S$ & 50.58 & - & - \\
\hline
\end{tabular}

${ }^{\mathrm{a}}$ Reference [7], ${ }^{\mathrm{b}}$ reference [10], ${ }^{\mathrm{c}}$ reference [11].

others theoretical calculation [10]. In addition, to reproduce the correct sign of the elastic constants, the value of Cauchy discrepancy $\left(\delta=C_{11}-C_{12}\right)$ have also been calculated. Due to including the charge transfer effect, the present model able to explain the Cauchy discrepancy $(\delta)$. The two-body potential model failed to explain the Cauchy discrepancy $(\delta)$. The value of pressure derivative of bulk modulus has been compared with the first principle calculation [11]. The present value of $d B_{T} / d P$ is comparable with the others result [11]. The value of shear modulus $(S)$ has also been calculated by the present model for $\mathrm{PbS}$. The value of $\mathrm{S}$ is given in Table 3.

\section{Conclusion}

In conclusion, we have applied Three Body Potential (TBP) model (including the charge transfer effect) to investigate the structural and mechanical properties of $\mathrm{PbS}$. The results are summarized as follows.

(1) The present compound crystallizes in six-foldcoordinated NaCl-type structure $\left(B_{1}\right)$ at normal conditions and under pressure, they transform to the eight-fold-coordinated CsCl-type structure $\left(B_{2}\right)$.

(2) During the $\mathrm{NaCl}$ to $\mathrm{CsCl}$ phase transition, the volume discontinuity in pressure volume phase diagram 
identifies the occurrence of first-order phase transition.

(3) The present calculated phase transition pressure and volume collapses are in general in good agreement with the available data.

(4) The calculated values of second-order elastic constants, their pressure derivatives, and Cauchy discrepancy $(\delta)$ are in general better than theoretical results and good agreement with the available experimental results.

(5) Our model able to explain the previous drawbacks of Cauchy discrepancy $(\delta)$ in the second-order elastic constants.

Finally, it may be concluded that there is generally a good agreement of three-body potential (TBP) model with the available experimental data than others theoretical values. Consequently the present model has successfully predicted the compression curves and phase diagrams giving the phase transition pressure, associated volume collapse correctly for the present compound. The present approach may also applied for other semiconductors of the same group. Moreover, the present model is able to explain the Cauchy discrepancy. The calculated values of Cauchy discrepancy $(\delta)$ have been given in Table 3 for the present compound.

\section{References}

[1] S. P. Zimin, E. S. Gorlachev, I. I. Amirov, H. Zogg, E. Ambramof, and P. H. O. Rappl, "Sputtering rates of lead chalcogenide-based ternary solid solutions during inductively coupled argon plasma treatment," Semicondutor Science \& Technology, vol. 26, Article ID 105003, 5 pages, 2011.

[2] S. Kumar and M. J. M. Khan, "Schottky barrier junctions of gold with lead chalcogenides: growth and characteristics," Chalcogenide Letters, vol. 9, no. 3, pp. 99-103, 2012.

[3] R. Dornhaus, G. Nimtz, and B. Schlicht, Narrow Gap Semiconductors, Springer, Berlin, Germany, 1985.

[4] T. Chattopadhyay, H. G. von Schnering, W. A. Grosshans, and W. B. Holzapfel, "High pressure X-ray diffraction study on the structural phase transitions in $\mathrm{PbS}, \mathrm{PbSe}$ and $\mathrm{PbTe}$ with synchrotron radiation," Physica $B+C$, vol. $139-140$, pp. 356360, 1986.

[5] J. Maclean, P. D. Hatton, R. O. Piltz, J. Crain, and R. J. Cernik, "Structural studies of semiconductors at very high pressures," Nuclear Instruments and Methods in Physics Research B, vol. 97, no. 1-4, pp. 354-357, 1995.

[6] A. Onodera, Y. Fujii, and S. Sugai, "Polymorphism and amorphism at high pressure," Physica $B+C$, vol. 139-140, pp. 240-245, 1986.

[7] O. Madelung, M. Schulz, and H. Weiss, Landolt-Bornstei, vol. 17 of New Series, Springer, Berlin, Germany, 1983.

[8] Y. Bencherif, A. Boukra, A. Zaoui, and M. Ferhat, "Highpressure phases of lead chalcogenides," Materials Chemistry and Physics, vol. 126, no. 3, pp. 707-710, 2011.

[9] J. Z. Jiang, L. Gerward, R. Secco, D. Frost, J. S. Olsen, and J. Truckenbrodt, "Phase transformation and conductivity in nanocrystal PbS under pressure," Journal of Applied Physics, vol. 87 , no. 5 , pp. $2658-2660,2000$.
[10] Y. Zhang, X. Ke, C. Chen, J. Yang, and P. R. C. Kent, "Thermodynamic properties of $\mathrm{PbTe}, \mathrm{PbSe}$, and $\mathrm{PbS}$ : firstprinciples study," Physical Review B, vol. 80, no. 2, Article ID 024304, 12 pages, 2009.

[11] P. Bhambhani, N. Munjal, G. Sharma, V. Vyas, B. K. Sharma, and B. K. : "First-principles study of B1 to B2 phase transition in PbS," Journal of Physics, vol. 377, Conference 1, Article ID 012068, 2012.

[12] C. E. Sims, G. D. Barrera, N. L. Allan, and W. C. Mackrodt, "Thermodynamics and mechanism of the B1-B2 phase transition in group-I halides and group-II oxides," Physical Review $B$, vol. 57, no. 18, pp. 11164-11172, 1998.

[13] S. Froyen and M. L. Cohen, "Structural properties of $\mathrm{NaCl}$ and $\mathrm{KCl}$ under pressure," Journal of Physics C, vol. 19, no. 15, pp. 2623-2632, 1986.

[14] R. K. Singh, "Many body interactions in binary ionic solids," Physics Reports, vol. 85, no. 5, pp. 259-401, 1982.

[15] P. Bhardwaj, S. Singh, and N. K. Gaur, "Structural and elastic properties of barium chalcogenides ( $\mathrm{BaX}, \mathrm{X}=\mathrm{O}, \mathrm{Se}, \mathrm{Te})$ under high pressure," Central European Journal of Physics, vol. 6, no. 2, pp. 223-229, 2008.

[16] P. Bhardwaj, S. Singh, and N. K. Gaur, "Structural, elastic and thermophysical properties of divalent metal oxides with $\mathrm{NaCl}$ structure," Materials Research Bulletin, vol. 44, no. 6, pp. 13661374, 2009.

[17] S. Singh and P. Bhardwaj, "Structural phase stability and elastic behavior of III-V scandium pnictides," Physica B, vol. 406, no. 8, pp. 1615-1621, 2011.

[18] P. Bhardwaj and S. Singh, "Structural analysis of partially covalent lanthanide and actinide bismuthides with phasetransition pressures below $14 \mathrm{GPa}$," Physica Status Solidi B, vol. 249, no. 1, pp. 38-49, 2012.

[19] P. Bhardwaj, "Structural and thermophysical properties of cadmium oxide," ISRN Thermodynamics, vol. 2012, Article ID 798140, 4 pages, 2012.

[20] P. Bhardwaj, "Structural study of transition metal carbides," Acta Physica Polonica A, vol. 122, no. 1, pp. 138-141, 2012. 

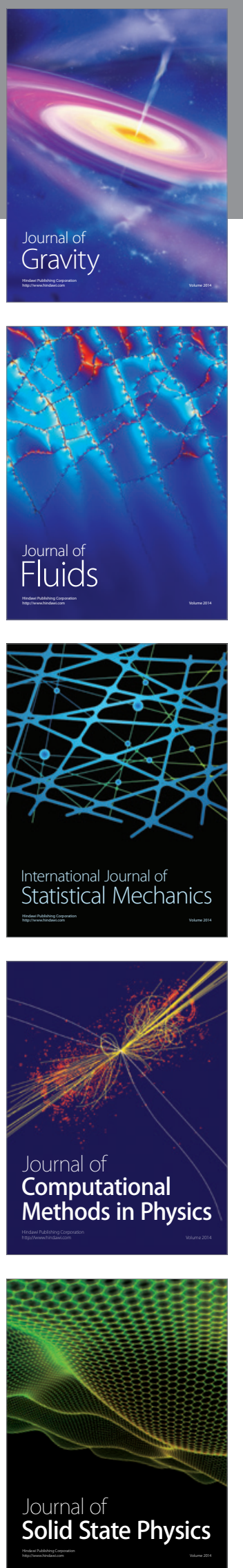
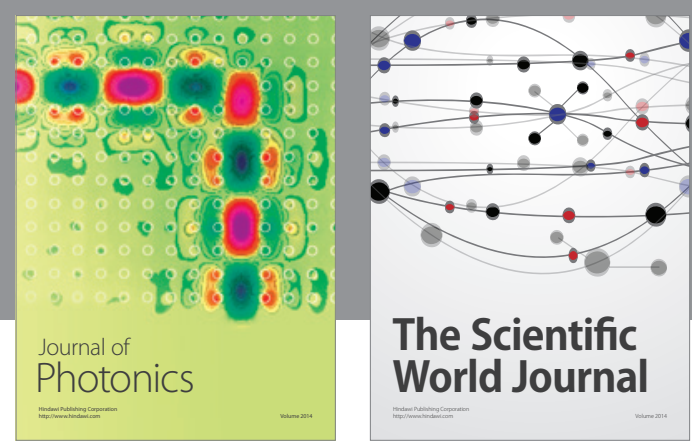

The Scientific World Journal

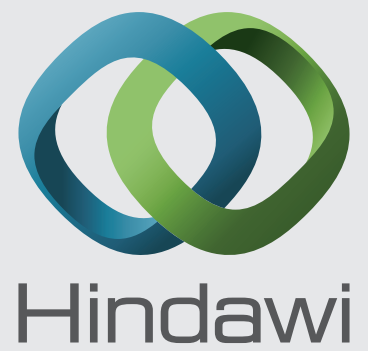

Submit your manuscripts at http://www.hindawi.com
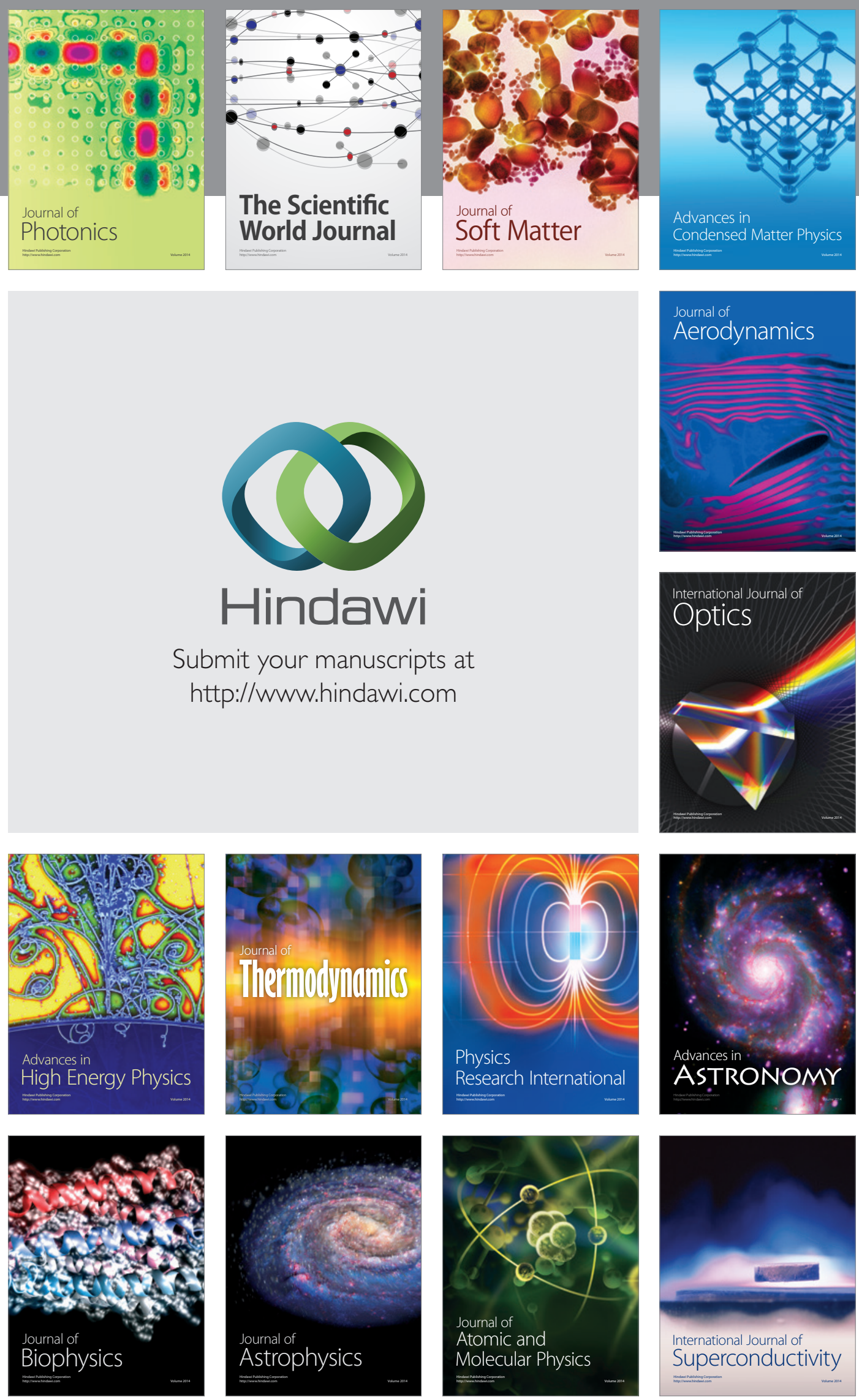
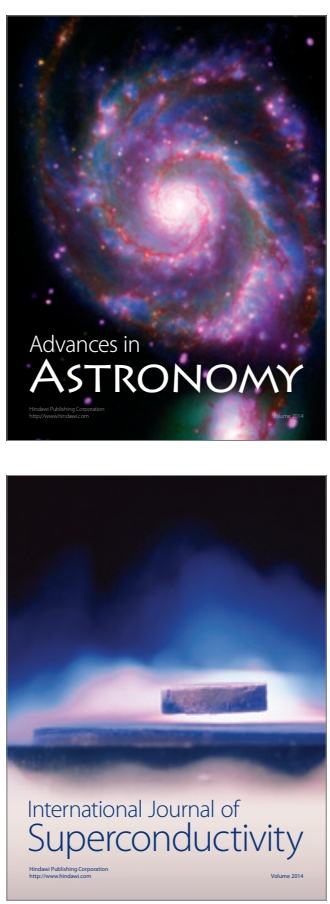